\title{
PREVALÊNCIA DE Dioctophyme renale (Goeze, 1782) EM CÃES DE UMA ORGANIZAÇÃO NÃO GOVERNAMENTAL DO SUL DO RIO GRANDE DO SUL - BRASIL
}

\author{
(Prevalence of Dioctophyme renale (Goeze, 1782) in dogs from a Non- \\ Governmental Organization of the south of Rio Grande do Sul - Brazil) \\ Pâmela Caye ${ }^{1}$, Tatiane Schmitt Tavares Novo ${ }^{1}$, Guilherme Albuquerque de Oliveira Cavalcanti ${ }^{1}$,
Josaine Cristina da Silva Rappeti \\ ${ }^{1}$ Universidade Federal de Pelotas, Pelotas, Rio Grande do Sul, Brasil
}

Corresponding author: pamiscaye@gmail.com

RESUMO: A dioctofimose é a doença causada por Dioctophyme renale (D. renale), um nematódeo de carnívoros, que afeta principalmente o rim direito dos animais. Raramente, pode afetar outros sítios anatômicos, como rim esquerdo, musculatura abdominal, vesícula urinária e, ainda, ser encontrado livre nas cavidades abdominal ou torácica. $\mathrm{O}$ agente é transmitido com o consumo do hospedeiro intermediário (Lumbriculus variegatus) e dos hospedeiros paratênicos (peixes, sapos e rãs). $O$ objetivo deste trabalho foi realizar uma investigação ultrassonográfica abdominal para pesquisar a prevalência de $D$. renale em cães de uma Organização Não Governamental (ONG) da região de Pelotas, no sul do estado do Rio Grande do Sul - Brasil, e avaliar alterações hematológicas, bioquímicas e urinárias nos pacientes positivos. De 63 cães examinados por ultrassonografia, seis apresentaram imagens compatíveis à presença de $D$. renale no rim direito e um na cavidade abdominal. No total, $11,11 \%(n=7)$ dos animais submetidos à ultrassonografia foram positivos para a presença de $D$. renale. Os animais tinham histórico de viver em ambiente precário, com uma superpopulação de cães sem acesso à água ou alimento adequado. Tais condições favorecem o ciclo biológico natural de $D$. renale, possibilitando a disseminação do parasito. É possível concluir que, além do método principal de diagnóstico, o exame do sedimento urinário, a ultrassonografia abdominal é um método complementar e eficaz na detecção de parasitismo por $D$. renale em animais assintomáticos e que vivem em locais de risco para a infecção, como apresentado neste trabalho.

Palavras-chave: Dioctofimose; rim; zoonose.

ABSTRACT: Dioctophymosis is a disease caused by Dioctophyme renale ( $D$. renale), a carnivorous nematode that mainly affects the right kidney of animals. It rarely affects other anatomic sites, such as the right kidney, abdominal musculature, urinary bladder, or it can be found freely in the abdominal and thoracic cavities. The agent is transmitted by the consumption of the intermediate host (Lumbriculus variegatus) and paratenic hosts (fishes, frogs and toads). This disease usually has a low casuistic in populations. The objective of this study was to carry out an ultrasonographic investigation on the prevalence of dioctophymosis in dogs of a Non-Governmental Organization in Pelotas region, in the south of the state of Rio Grande do Sul - Brazil, and to evaluate hematological, biochemical and urinary alterations in positive patients. Of 63 dogs examined by ultrasound, six showed images compatible with the presence of $D$. renale in the right kidney and one patient presented the parasite released in the abdominal cavity. Altogether, $11,11 \%(n=7)$ of 
the studied animals were positive for the presence of $D$. renale. The animals had a history of living in a precarious ambient, with a super population of dogs without access to water or appropriated food. Such conditions favour natural biological cycle of D. renale, allowing the spread of the parasite. It is possible to conclude that, in addition to the main diagnostic method, the examination of urinary sediment, abdominal ultrasound is a complementary and effective method for the detection of parasitism by $D$. renale in asymptomatic animals that live in places at risk for infection, such as presented in this work.

Keywords: Dioctophymosis; kidney; zoonosis. 
INTRODUÇÃO

A dioctofimose é uma doença causada por Dioctophyme renale (Goeze, 1782), conhecido como verme gigante do rim. É um nematódeo que se localiza, principalmente, nos rins dos hospedeiros, com maior prevalência no rim direito (Measures, 2001). Localizações menos comuns já foram relatadas na literatura, como rim esquerdo (Pedrassani et al., 2010; Perera et al., 2017b), tumor em glândula mamária inguinal (Sousa et al., 2011), útero gravídico (Veiga et al., 2012), subcutâneo (Silveira et al., 2015) e musculatura abdominal (Caye et al., 2018).

Os animais com o parasitismo renal geralmente são assintomáticos, podendo em casos sintomáticos apresentar fraqueza, ascite, dor à palpação abdominal, polaquiúria, disúria e anorexia. Nas análises laboratoriais, as alterações mais frequentes são anemia, uremia, hematúria, proteinúria e presença de ovos em sedimento urinário (Measures, 2001; Pedrassani e Nascimento, 2015).

A parasitose pode ser diagnosticada com realização de exames complementares de imagem, por meio de radiografia e ultrassonografia (Measures, 2001; Silveira et al., 2015). O exame laboratorial de sedimento urinário permite o diagnóstico por meio da visualização dos ovos pela microscopia (Silveira et al., 2015).

Presente em diversas regiões do mundo, o parasitismo já foi descrito em países como Brasil, Uruguai, Paraguai, Colômbia, México, Estados Unidos, Canadá (Paras et al., 2018), China, Indonésia, Grécia e Austrália (Yang et al., 2019). O parasito infecta, principalmente, carnívoros por ingestão do hospedeiro intermediário, o anelídeo oligoqueta Lumbriculus variegatus, e por predação de hospedeiros paratênicos, como peixes e anfíbios. O parasito infecta várias espécies animais, incluindo o ser humano, lontras (Lontra longicaudis), lobos (Canis lupus), cães (Canis familiaris), gatos (Felis catus), furões (Galictis cuja) e visons (Mustela vison), sendo os últimos considerados reservatórios do parasito (Pedrassani e Nascimento, 2015).

Cães errantes com alimentação não seletiva, próximos a coleções de água, são mais predispostos ao desenvolvimento da doença, porque um ambiente aquático é necessário para o desenvolvimento do hospedeiro intermediário, com consequente evolução do ciclo biológico do parasito (Kommers et al., 1999; Pedrassani e Nascimento, 2015). Os cães errantes tendem a consumir água não tratada e restos de peixes, além de desenvolver comportamento predatório com sapos e rãs, tendo maiores riscos de infecção, uma vez que estes são definidos como hospedeiros paratênicos (Pedrassani et al., 2010; Silveira et al., 2015). A superfície do solo contaminada com ovos, inundações e presença de hospedeiros intermediários e definitivos favorecem a disseminação do parasito, o que pode explicar os diferentes dados de prevalência de acordo com a região estudada (Burgos el al., 2014).

A dioctofimose costuma apresentar casuísticas muito distintas em determinadas populações estudadas. Kommers et al. (1999), em estudo com 3.259 cães necropsiados, encontrou uma prevalência de $0,49 \% \quad(\mathrm{~N}=16)$, sendo 12 animais parasitados não domiciliados. Já o estudo de Leite et al. (2005), com achados clínicos de dois cães e necropsia de outros 1958, determinou uma prevalência de $0,56 \%$ de dioctofimose na cidade de Curitiba $\mathrm{PR}$, sem considerar a origem dos animais. Burgos et al. (2014) encontraram uma prevalência de $42,1 \%$ na análise urinária de 171 cães da região de La Plata - Argentina. Pedrassani et al. (2017) avaliaram 197 
cães, em Três Barras - Santa Catarina, por meio de urinálise e encontraram $14,2 \%$ dos animais parasitados com $D$. renale.

O presente trabalho objetivou analisar a prevalência de $D$. renale em cães oriundos de uma ONG, localizada na região de Pelotas - Rio Grande do Sul, pelo exame de ultrassonografia abdominal, e avaliar as alterações hematológica, bioquímica e urinária dos pacientes positivos.

\section{MATERIAL E MÉTODOS}

A população alvo do estudo foram cães de uma ONG da região de Pelotas - Rio Grande do Sul, com histórico de terem pertencido a um acumulador de animais, onde mantinha mais de 200 cães em condições insalubres. Os cães foram transferidos para uma fazenda, sede da ONG. Nove meses após a transferência, 63 animais foram selecionados aleatoriamente para participarem do presente estudo.

Encaminhados ao Hospital de Clínicas Veterinárias da Universidade Federal de Pelotas (HCV - UFPel), os pacientes foram submetidos aos exames clínico, hematológico, urinário e ultrassonográfico abdominal. Os resultados de hematologia e bioquímica sérica foram avaliados de acordo com as referências para a espécie definidas por Weiss e Wardrop (2010) e Kaneko et al. (2008), respectivamente. Foram coletados também dados como sexo (fêmea ou macho), raça (SRD - sem raça definida ou $R D$ - raça definida) e idade (jovem, adulto ou idoso). A idade foi definida de acordo com a dentição, sendo classificados como jovens os cães com dentição decídua, adultos com dentição permanente íntegra e idosos aqueles com desgaste considerável dos incisivos (König e Liebich, 2011).
Para o exame ultrassonográfico, os pacientes foram contidos fisicamente em decúbito dorsal e tricotomizados em região abdominal. A avaliação ultrassonográfica da cavidade abdominal foi realizada utilizando-se um aparelho de ultrassonografia com transdutor multifrequencial microconvexo 8C-RS (6-10 $\mathrm{MHz})$ e transdutor multifrequencial linear 12LRS (7-12 MHz). Durante a realização da ultrassonografia, a urina dos animais foi coletada por cistocentese guiada pelo aparelho ultrassonográfico. Em seguida, amostra de sangue foi coletada por meio de punção da veia jugular e armazenada em frascos sem e com anticoagulante (EDTA). Os animais diagnosticados para presença de $D$. renale foram encaminhados para o setor de clínica cirúrgica do HCV - UFPel para realizar o procedimento cirúrgico de retirada do parasito.

\section{RESULTADOS}

Do total de 63 cães examinados, $37(58,73 \%)$ eram fêmeas e 26 $(41,26 \%)$ eram machos. Sobre os dados coletados acerca da raça, 60 (95,23\%) animais foram classificados como Sem Raça Definida (SRD). Três animais eram de raça definida (RD), sendo um Dálmata, um Schnauzer e um Pastor Alemão. Em relação à faixa etária, 30 $(48,40 \%)$ animais eram idosos, 25 $(39,10 \%)$ eram adultos e oito $(12,50 \%)$ jovens.

Um total de sete cães foi diagnosticado com o parasitismo, representando uma prevalência de $11,11 \%$ na população estudada, sendo seis em rim direito e um livre em cavidade abdominal. Dos animais parasitados, quatro eram fêmeas e três machos, sendo seis adultos e um jovem e todos eram SRD.

Os exames hematológicos dos animais com $D$. renale revelaram três 
pacientes com eosinofilia, sendo que destes, um também apresentava leucocitose com desvio à esquerda e, outro, anemia discreta com $32,4 \%$ de hematócrito (Referência: 37 - 55\%, conforme Weiss e Wardrop, 2010). Os valores de bioquímica sanguínea não apresentaram alterações nos padrões de ureia e creatinina. Os seis pacientes com o parasito no rim direito apresentaram hematúria, sendo que três destes continham ovos de $D$. renale e um apresentava leucocitúria. Os resultados obtidos estão relacionados na Tabela 1.

Tabela 1. Perfil e alterações laboratoriais dos cães parasitados por Dioctophyme renale oriundos de uma Organização Não Governamental do sul do Rio Grande do Sul.

\begin{tabular}{|c|c|c|c|c|c|c|c|c|c|c|c|}
\hline \multirow[b]{2}{*}{ Cão } & \multirow[b]{2}{*}{ Idade } & \multirow[b]{2}{*}{ Sexo } & \multirow[b]{2}{*}{ Raça } & \multicolumn{3}{|c|}{ Hematologia } & \multirow[b]{2}{*}{ Bioquímica } & \multicolumn{3}{|c|}{ Urinálise } & \multirow[b]{2}{*}{$\begin{array}{l}\text { Localização } \\
\text { anatômica }\end{array}$} \\
\hline & & & & Anemia & LDE & Eosinofilia & & Hematúria & Leucocitúria & $\begin{array}{l}\text { Ovos } \\
\text { de } D \text {. } \\
\text { renale }\end{array}$ & \\
\hline 1 & Adulto & M & SRD & - & - & - & S/A & $\mathbf{x}$ & - & $x$ & RD \\
\hline 2 & Adulto & M & SRD & - & $x$ & $x$ & S/A & $\mathbf{x}$ & - & $x$ & $\mathrm{RD}$ \\
\hline 3 & Adulto & M & SRD & $x$ & - & $x$ & $S / A$ & $x$ & - & - & $\mathrm{RD}$ \\
\hline 4 & Jovem & $\mathrm{F}$ & SRD & - & - & - & S/A & $x$ & - & - & RD \\
\hline 5 & Adulto & $\mathrm{F}$ & SRD & - & - & $x$ & S/A & $\mathbf{x}$ & - & $x$ & $\mathrm{RD}$ \\
\hline 6 & Adulto & $\mathrm{F}$ & SRD & - & - & - & S/A & $x$ & $x$ & - & RD \\
\hline 7 & Adulto & $\mathrm{F}$ & SRD & - & - & - & S/A & - & - & - & $\mathrm{CA}$ \\
\hline
\end{tabular}

As avaliações ultrassonográficas permitiram diagnosticar o parasito no rim direito em seis pacientes (Figura 1). No sétimo paciente, um exemplar de $D$. renale foi encontrado na cavidade abdominal, igualmente detectado por meio do exame ultrassonográfico, sem alterações renais visíveis. Os cães positivos foram encaminhados para o centro cirúrgico para a retirada dos parasitos.

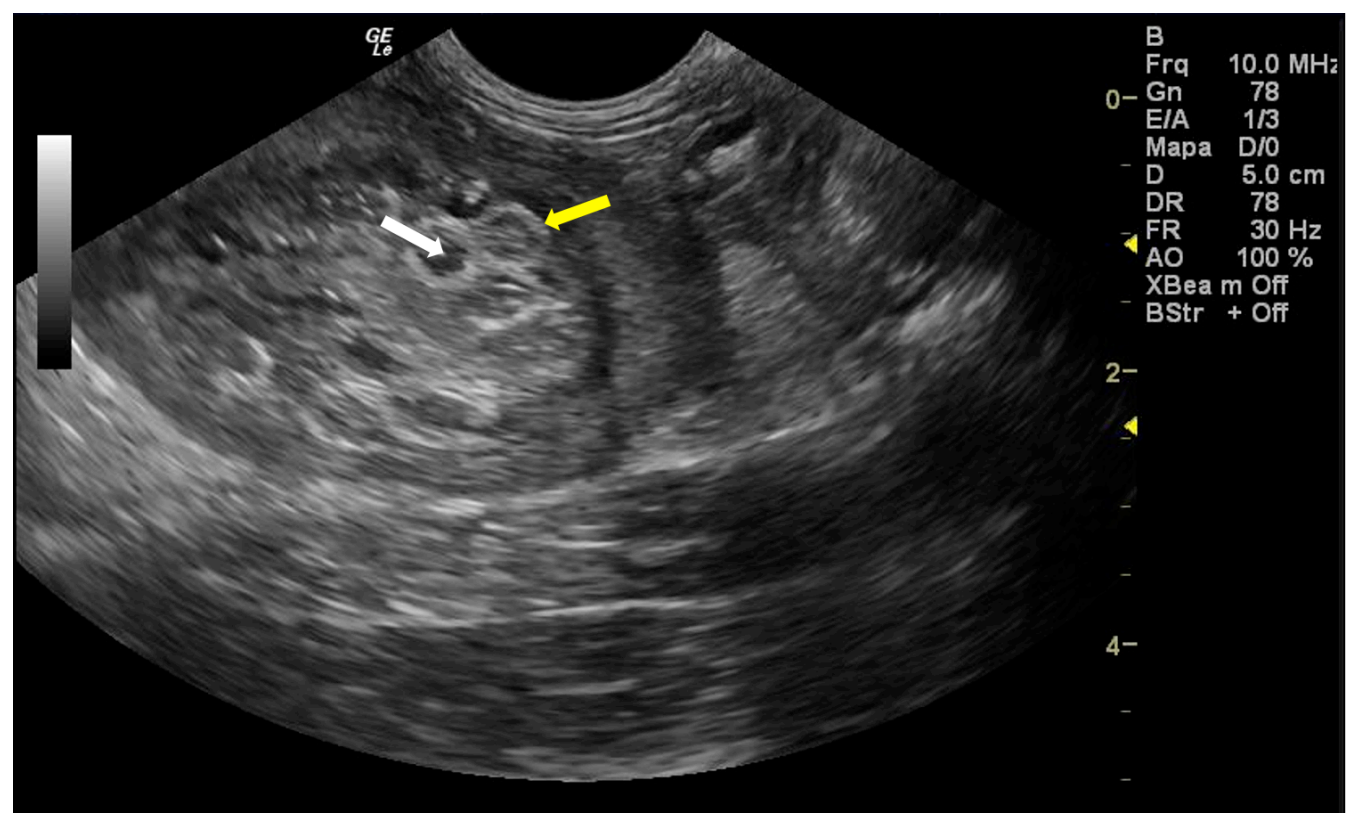

Figura 1. Imagens compatíveis com Dioctophyme renale no interior do rim direito de um cão. As setas indicam cortes transversais do parasito, com bordas hiperecóicas (seta amarela) e centro hipoecóico (seta branca).

Os procedimentos cirúrgicos uma nefrotomia para os pacientes com realizados foram cinco nefrectomias e o parasitismo renal. Uma laparotomia 
exploratória foi realizada no paciente com o parasito livre na cavidade abdominal, detectado por ultrassonografia. Foram obtidos um total de quatro exemplares machos e oito exemplares fêmeas de $D$. renale, com sua diferenciação sexual baseada na respectiva presença ou ausência de bolsa copuladora na porção terminal do corpo (Radman et al., 2017). Todos os parasitos foram medidos e o maior entre os machos mediu $24,5 \mathrm{~cm}$ de comprimento e, entre as fêmeas, $67 \mathrm{~cm}$ (Figura 2).

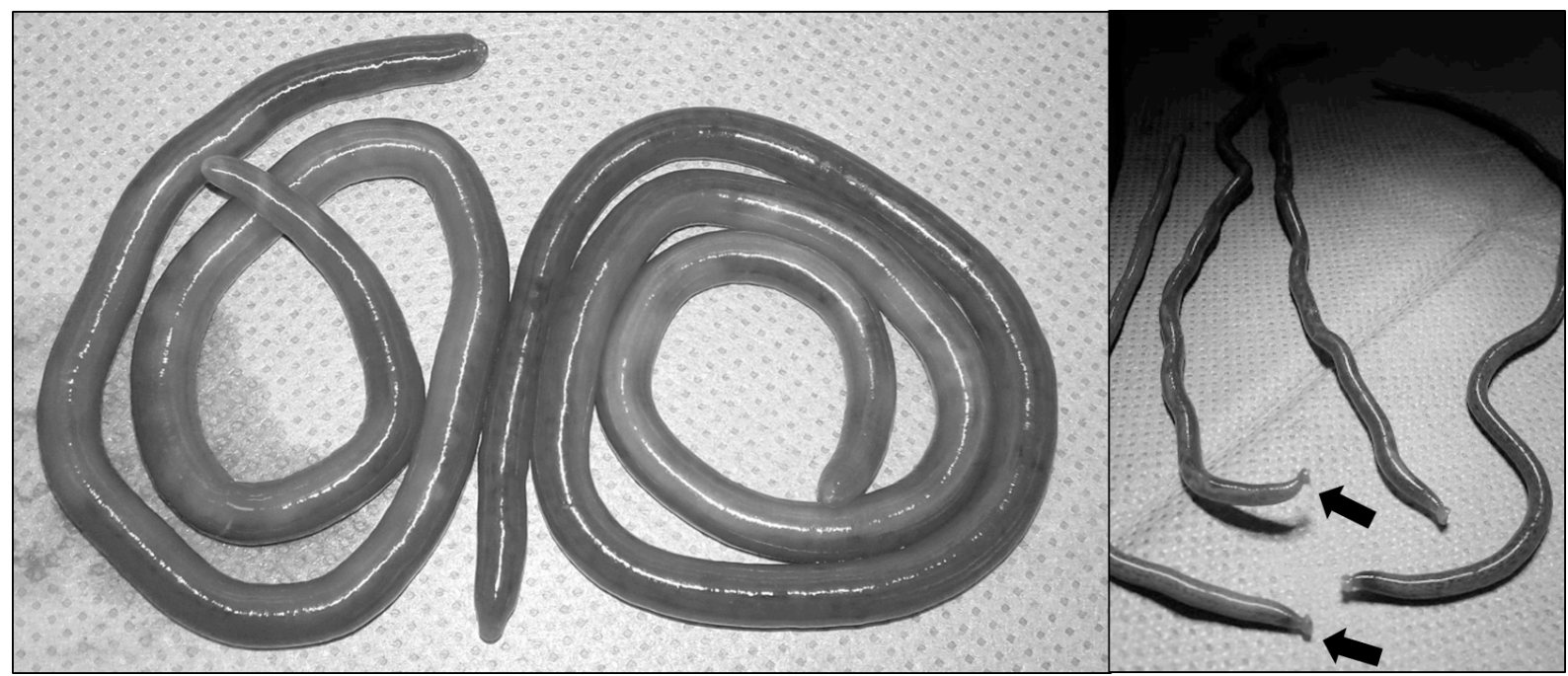

Figura 2. Exemplares de Dioctophyme renale removidos cirurgicamente do interior renal de cães de uma ONG do sul do Rio Grande do Sul. A - Fêmeas. B - Machos. Observar a bolsa copuladora em formato de campânula na porção terminal dos machos (setas pretas).

\section{DISCUSSÃO}

Antes do acolhimento pela ONG, os animais encontravam-se em um local impróprio, sem saneamento, acesso à agua tratada e nem alimentação em quantidade e qualidade adequadas, características que favorecem a disseminação da doença (Mace e Anderson, 1975; Burgos et al., 2014). Submetidos à péssima qualidade de vida, acredita-se que os animais tenham ingerido água de esgoto, consumido alimento impróprio e desenvolvido hábitos predatórios. A predação de peixes, rãs e sapos, albergando as formas larvais do parasito, é uma das formas de infecção por $D$. renale. Ocorre, também, quando há ingestão do hospedeiro intermediário, o anelídeo oligoqueta aquático Lumbriculus variegatus, que se desenvolve no sedimento de rios, lagos e córregos de água doce (Measures, 2001; Pedrassani e Nascimento, 2015).
Nas instalações da ONG, os cães foram acomodados em canis em grupos de cinco a dez animais. A alimentação instituída foi ração comercial para cães e água potável sempre disponível. Por se tratar de uma grande área rural, não houve controle sobre possíveis hábitos predatórios terem ocorrido também nas suas dependências.

Estudos demonstram que o nematódeo infecta, principalmente, cães adultos e sem raça definida (Cottar et al., 2012; Silveira et al., 2015). Análises epidemiológicas associaram a infecção por $D$. renale e o acesso à rua ou a jardins e áreas rurais (Kommers et al., 1999; Cottar et al., 2012; Silveira et al., 2015). Isso acontece em decorrência da disseminação de ovos de $D$. renale no solo, por meio da urina de cães infectados, e da presença do hospedeiro intermediário, que se desenvolve em ambientes aquáticos (Burgos et al., 2014). 
A região da cidade de Pelotas, no sul do Rio Grande do Sul, apresenta condições favoráveis para o desenvolvimento de $D$. renale, como rios, inundações, presença de ovos do parasito no solo (Perera et al., 2017a) e cães com hábitos alimentares de risco (Burgos et al., 2014), aumentando as chances de ingestão de hospedeiros paratênicos e intermediários infectados com larvas de $D$. renale. Os dados de ocorrência mostram que a região apresenta uma alta prevalência de parasitismo por $D$. renale, onde Rappeti et al. (2016) relataram 95 casos do parasitismo em cães e gatos, em um estudo retrospectivo de diagnósticos realizados por meio de necropsias e ultrassonografias.

O período pré-patente de $D$. renale pode variar de 135 dias (Mace e Anderson, 1975) até 5 a 6 meses (Acha e Szyfres, 2003). Visto que os animais permaneceram na ONG por nove meses entre o resgate e o desenvolvimento do presente estudo, não é possível a exata definição do momento em que ocorreu a infecção, uma vez que ambos os ambientes, tanto antes quanto após o resgate, são favoráveis ao desenvolvimento do parasito.

Os resultados de exames laboratoriais obtidos foram semelhantes aos de Mesquita et al. (2014), que encontraram $75 \%$ dos cães com altas taxas de hematúria microscópica (6/8) e presença de ovos de $D$. renale no sedimento urinário de $62,5 \%$ (5/8) dos animais. A hematúria se mostra um sinal importante ao clínico veterinário na avaliação de cães com suspeita ao parasitismo. Da mesma forma, foram compatíveis os achados hematológicos, com apenas um cão apresentando anemia por queda na dosagem de hemoglobina (Mesquita et al., 2014). Nenhum paciente apresentou alterações de ureia e creatinina sérica, o que é explicado pelo fato de o rim contralateral comumente compensar o rim afetado (Measures, 2001).

O rim direito é o órgão mais afetado pela tendência migratória das larvas do parasito, levando à completa destruição renal (Kommers et al., 1999; Sapin et al., 2017). A rota de migração larval é alvo de diversos questionamentos, sendo que Hallberg (1953) encontrou indícios de que a mesma ocorre no ponto de íntimo contato entre o duodeno e o rim direito. Já Mace e Anderson (1975) indicaram que a migração das larvas ocorre através da parede gástrica, passando pela cavidade abdominal e fígado, antes de penetrarem no rim direito. Os autores concordam que a disposição anatômica dos órgãos influencia o local do parasitismo (Hallberg, 1953; Mace e Anderson, 1975).

Existem relatos de diagnóstico ultrassonográfico de parasitismo por $D$. renale em regiões anatômicas distintas, como livres na cavidade abdominal mesogástrica e epigástrica, na região inguinal, em subcutâneo paraprepucial (Silveira et al., 2015) e até mesmo em cavidade torácica (Mistieri et al., 2019) e no interior de útero gravídico (Veiga et al., 2012). Neste trabalho, três dos seis pacientes atendidos, com diagnóstico positivo em rim direito pela ultrassonografia, estavam eliminando ovos pela urina (Tabela 1). Isso mostra que o exame ultrassonográfico foi essencial para o diagnóstico dos outros três animais.

Os ovos observados no sedimento urinário eram acastanhados, com casca espessa e bioperculados, com até 80 $\mu \mathrm{m}$ de comprimento. Quando ausentes no sedimento urinário, o parasitismo não pode ser descartado, pois pode haver presença unicamente de parasitos machos no interior renal, de fêmeas imaturas ou parasitos presentes em outras regiões anatômicas do animal (Measures, 2001; Pedrassani et al., 
2010; Pedrassani e Nascimento, 2015; Silveira et al., 2015).

A ultrassonografia abdominal foi igualmente importante quando se considera que um dos pacientes apresentava apenas um parasito livre na cavidade abdominal, sem alterações renais ou das vias urinárias (ureteres, vesícula urinária e uretra). Logo, independente do sexo do parasito e da eliminação de ovos pelo mesmo, ovos jamais seriam excretados pela urina, não permitindo o diagnóstico através do exame de sedimento urinário e levando a um resultado falso-negativo (Measures, 2001).

Nos animais parasitados, a ultrassonografia permite visualizar as estruturas dos nematódeos em planos longitudinais e transversais. No plano longitudinal os parasitos são alongados e com paredes hiperecóicas, já no plano transversal as imagens obtidas se apresentam como halos hiperecóicos. Ambas as imagens apresentam interior hipoecóico (Pedrassani et al., 2010; Silveira et al., 2015).

Em alguns casos, a motilidade parasitária no interior do órgão pode auxiliar no diagnóstico. Quando há alta carga parasitária renal, a identificação das estruturas ultrassonográficas características pode ser prejudicada, como no caso descrito por Pedrassani et al. (2010), onde oito parasitos estavam no interior da cápsula renal, dificultando o diagnóstico. Neste mesmo caso, a avaliação de sedimento urinário apresentou resultados negativos, o que foi explicado pelo fato dos parasitos encontrados serem imaturos.

Os resultados deste trabalho mostraram que a ultrassonografia abdominal foi eficaz para o diagnóstico de $D$. renale presente nos rins $\mathrm{e}$ cavidade abdominal. Portanto, é um método que deve ser adotado na rotina clínica em animais oriundos de regiões endêmicas para a parasitose, permitindo um diagnóstico e tratamento precoce dos pacientes.

\section{CONCLUSÕES}

É possível concluir que o exame ultrassonográfico é um método que se mostrou eficaz no diagnóstico do parasitismo por $D$. renale mesmo em cães assintomáticos e negativos para detecção de ovos. Além disso, os fatores de risco, como animais oriundos de regiões endêmicas, situações que favorecem a infecção e alterações laboratoriais compatíveis com a infecção devem ser considerados para solicitar a ultrassonografia.

\section{NOTAS INFORMATIVAS}

O presente estudo teve os seus métodos utilizados aprovados pela Comissão de Ética em Utilização Animal (CEEA) da Universidade Federal de Pelotas, de acordo com as normas do CONCEA, sob o número de protocolo 8663.

\section{REFERÊNCIAS}

ACHA, P.N.; SZYFRES, B. Zoonosis y enfermedades transmisibles communes al hombre $y$ a los animales. 3. ed. Washington DC: Organización Panamericana de la Salud, 2003. 413p.

BURGOS, L.; ACOSTA, R.M.; FONROUGE, R.D. et al. Prevalence of a zoonotic parasite, Dioctophyma renale (Goeze, 1782), among male canines in a wild riverside area of La Plata river, province of Buenos Aires of Argentina. Revista de Patologia Tropical, v.43, n.4, p.420-426, 2014.

CAYE, P.; MILECH, V.; de LIMA, C.S. et al. Intramuscular Dioctophyme renale surgically removed from dog - rare case report. Scholars Journal of 
Agriculture and Veterinary Science, v.5, n.5, p.266-269, 2018.

COTTAR, B.H.; DITTRICH, G.; FERREIRA, A.A. et al. Achados ultrassonográficos de cães parasitados por Dioctophyma renale - Estudo retrospectivo. Veterinária e Zootecnia, v.19, n.1, p.8-11, 2012.

HALLBERG, C.W. Dioctophyma renale (Goeze, 1782) a study of the migration routes to the kidneys of mammals and resultant pathology. American Microscopical Society, v.72, n.4, p.351-363, 1953.

KANEKO, J.; HARVEY, J.; BRUSS, M. Clinical biochemistry of domestic animals. Cambridge: Academic Press, 2008. 928p.

KOMMERS, G.D.; ILHA, M.R.S.; BARROS, C.S.L. Dioctofimose em cães: 16 casos. Ciência Rural, v.29, n.3, p.517-522, 1999.

KÖNIG, H. E.; LIEBICH, H. Anatomia dos animais domésticos. $4^{\mathrm{a}}$ ed. Porto Alegre: Artmed, 2011. 788p.

LEITE, L.C.; CÍRIO, S.M.; DINIZ, J.M.F. et al. Lesões anatomopatológicas presentes na infecção por Dioctophyma renale (Goeze, 1782) em cães domésticos (Canis familiaris, Linnaeus, 1758). Archives of Veterinary Science, v.10, n.1, p.95-101, 2005.

MACE, T.F.; ANDERSON, R.C. Development of the giant kidney worm, Dioctophyma renale (Goeze, 1782) (Nematoda: Dioctophymatoidea). Canadian Journal of Zoology, v.53, p.1552-1568, 1975.

MEASURES, L.N. Dioctophymatosis. In: SAMUEL, W.M.; PYBUS, M.J.; KOCAN, A.A. Parasitic Diseases of Wild Mammals. Ames: lowa State University Press; 2001, Cap.13, p.357-364.

MESQUITA, L.R.; RAHAL, S.C.; FARIA, L.G. et al. Pre- and post-operative evaluations of eight dogs following right nephrectomy due to Dioctophyma renale. Veterinary Quarterly, v.34, n.3, p.167-171, 2014.

$\begin{array}{llr}\text { MISTIERI, M.L.A.; } & \text { DILL, } & \text { S.W.; } \\ \text { RAMPELOTTO, } & \text { C. } & \text { al. }\end{array}$ Dioctophymatosis as cause of dyspnea in a dog. Ciência Rural, v.49, n.1, p.1-5, 2019.

PARAS, K.L.; MILLER, L.; VEROCAI, G.G. Ectopic infection by Dioctophyme renale in a dog from Georgia, USA, and a review of cases of ectopic dioctophymosis in companion animals in the Americas. Veterinary Parasitology, v.14, p.111-116, 2018.

PEDRASSANI, D.; NASCIMENTO, A.A. Verme gigante renal. Revista Portuguesa de Ciências Veterinárias, v.110, n.593-594, p.30-37, 2015.

PEDRASSANI, D.; NASCIMENTO, A.A.; ANDRÉ, M.R. et al. Dioctophyme renale: prevalence and risk factors of parasitism in dogs of São Cristóvão district, Três Barras County, Santa Catarina, Brasil. Brazilian Journal of Veterinary Parasitology, v.26, n.1, p.39-46, 2017.

PEDRASSANI, D.; PILATI, C.; WENDT, S.B.T. et al. Diagnóstico ultrassonográfico de infecção intensa por Dioctophyme renale em rim esquerdo de cão - relato de caso. Clínica Veterinária, v.85, p.74-80, 2010.

PERERA, S.C.; CAPELLA, G.A.; PINTO, N.B. et al. First isolation of Dioctophyme renale eggs from an urban environment and identification of those from animal urine. Brazilian Journal of Veterinary Parasitology, v.26, n.1, p.89-91, 2017a.

PERERA, S.C.; RAPPETI, J.C.S.; MILECH, V. et al. Eliminação de Dioctophyme renale pela urina em canino com dioctofimatose em rim esquerdo e cavidade abdominal Primeiro relato no Rio Grande do Sul. Arquivo Brasileiro de Medicina 
Veterinária e Zootecnia, v.69, n.3, p.618-622, 2017b.

RADMAN, N.E.; GAMBOA, M.I.; BUTTI, M.J. et al. Ocurrence of dioctophymosis in canines within a riparian zone of the Río de La Plata watercourse, in Ensenada, Buenos Aires Province, Argentina. Veterinary Parasitology: Regional Studies and Reports, v.10, p.43-50, 2017.

RAPPETI, J.C.S.; MASCARENHAS, C.S.; PERERA, S.C. Dioctophyme renale (Nematoda: Enoplida) in domestic dogs and cats in the extreme south of Brazil. Brazilian Journal of Veterinary Parasitology, v.26, n.1, 2016.

SAPIN, C.F.; SILVA-MARIANO, L.C.; PIOVESAN, A.D. et al. Estudo anatomopatológico de rins parasitados por Dioctophyme renale em cães. Acta Scientiae Veterinariae, v.45, n.1441, 2017.

SILVEIRA, C.S.; DIEFENBACH, A.; MISTIERI, M.L. et al. Dioctophyma renale em 28 cães: aspectos clinicopatológicos e ultrassonográficos. Pesquisa Veterinária Brasileira, v.35, n.11, p.899-905, 2015.

SOUSA, A.A.R.; SOUSA, A.A.S.; COELHO, M.C.O.C. et al. Dioctofimose em cães. Acta Scientiae Veterinariae, v.39, n.3, p.1-4, 2011.

VEIGA, C.C.P.; OLIVEIRA, P.C.; FERREIRA, A.M.R. et al. Dioctofimose em útero gravídico em cão - relato de caso. Revista Brasileira de Medicina Veterinária, v. 34, n.3, p.188-191, 2012.

WEISS, D.J.; WARDROP, K.J. Schalm's Veterinary Hematology. lowa: Blackwell Publishing, 2010. 1232p.

YANG, F.; ZHANG, W.; GONG, B. et al. A human case of Dioctophyma renale (giant kidney worm) accompanied by renal câncer and a retrospective study of dioctophymiasis. Parasite, v.26, n.22, 2019. 